\title{
Blood Pressure Assessment with Differential Pulse Transit Time and Deep Learning: A Proof of Concept
}

\author{
Vicent Ribas Ripoll ${ }^{\mathrm{a}} \quad$ Alfredo Vellido $^{\mathrm{b}}$ \\ ${ }^{a}$ Eurecat, Centre Tecnològic de Catalunya, eHealth Unit, Barcelona, Spain; ${ }^{b}$ Intelligent Data Science and Artificial \\ Intelligence (IDEAI) Research Center, Universitat Politècnica de Catalunya (UPC BarcelonaTech), Barcelona, Spain
}

\section{Keywords}

Deep learning $\cdot$ Restricted Boltzmann machines ·

Hemodynamic monitoring · Pulse transit time

\begin{abstract}
Background: Modern clinical environments are laden with technology devices continuously gathering physiological data from patients. This is especially true in critical care environments, where life-saving decisions may have to be made on the basis of signals from monitoring devices. Hemodynamic monitoring is essential in dialysis, surgery, and in critically ill patients. For the most severe patients, blood pressure is normally assessed through a catheter, which is an invasive procedure that may result in adverse effects. Blood pressure can also be monitored noninvasively through different methods and these data can be used for the continuous assessment of pressure using machine learning methods. Previous studies have found pulse transit time to be related to blood pressure. In this short paper, we propose to study the feasibility of implementing a data-driven model based on restricted Boltzmann machine artificial neural networks, delivering a first proof of concept for the validity and viability of a method for blood pressure prediction based on
\end{abstract}

\section{KARGER}

(C) 2018 S. Karger AG, Basel

E-Mail karger@karger.com

www.karger.com/kdd these models. Summary and Key Messages: For the most severe patients (e.g., dialysis, surgery, and the critically ill), blood pressure is normally assessed through invasive catheters. Alternatively, noninvasive methods have also been developed for its monitorization. Data obtained from noninvasive measurements can be used for the continuous assessment of pressure using machine learning methods. In this study, a restricted Boltzmann machine artificial neural network is used to present a first proof of concept for the validity and viability of a method for blood pressure prediction.

(c) 2018 S. Karger AG, Basel

\section{Introduction}

Hemodynamic monitoring is essential in dialysis, surgery, and in critically ill patients. For the most severe patients, blood pressure (BP) is normally assessed through a catheter inserted in a peripheral artery, most common-

Contribution from the 2nd meeting of "Science for Dialysis", organized at the University Hospital of Bellvitge, L'Hospitalet de Llobregat, Barcelona, Spain, on September 28, 2018. 


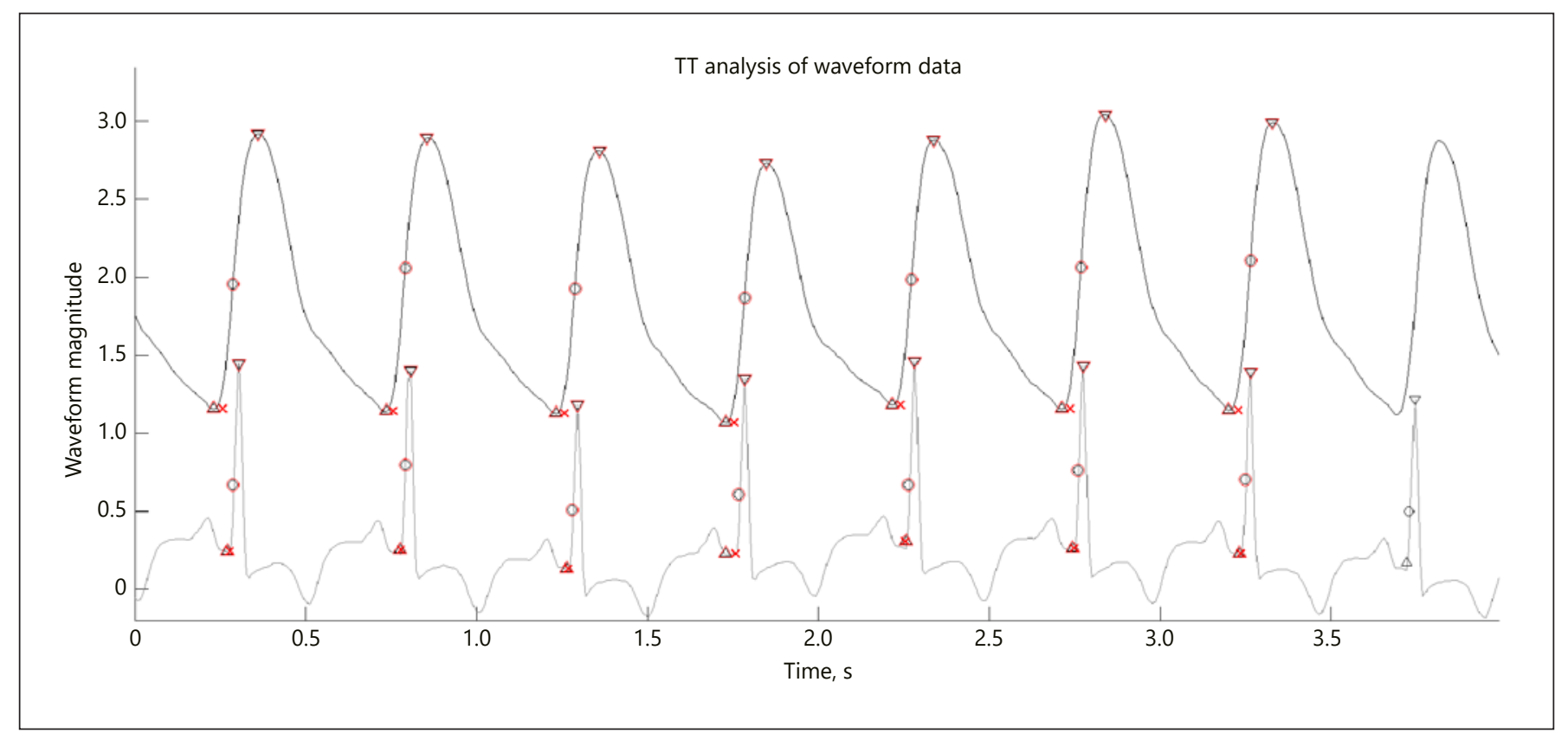

Fig. 1. Pulse transit time between arterial pressure signals and photoplethysmography.

ly in radial or femoral arteries and less frequently in axillar and pedal arteries. Arterial cannulation is an invasive procedure with the potential to result in adverse effects such as infection or thrombosis, which are associated with increased mortality and costs [1].

$\mathrm{BP}$ can also be monitored noninvasively through different methods. Classically, BP has been assessed through the auscultatory method based on the sound of the brachial artery pulse when an arm cuff is deflated (Korotkoff sounds). The oscillometric method is the most common method for automatic medical devices [2]. This method also uses an inflatable cuff with a pressure sensor inside it. Other methods include arterial tonometry [3], which places a tonometer perpendicular to an artery (normally, the radial artery), whilst most of the methods using photoplethysmography (PPG) also resort to a cuff placed in one or two fingers for the assessment of blood volume (BV). In this approach, transmural pressure is estimated through oscillometry [2]. The cuff pressure is continually varied to maintain this BV throughout a cardiac cycle via a servomechanism. Therefore, the applied pressure to maintain BV must be equal to BP.

Therefore, most BP assessment methods available for clinical practice require a cuff, which is cumbersome and, in some cases, like in volume clamping, painful. Moreover, cuff-based methods have been associated with underestimation of systolic blood pressure (SBP) and over- estimation of diastolic blood pressure (DBP), especially in obese patients and in the presence of arrhythmia $[4,5]$.

Other methods for the noninvasive assessment of BP use machine learning techniques to exploit the correlation between the PPG and arterial pressure (ART) signals. For example, Ruiz-Rodríguez et al. [6] proposes a datadriven model created with deep learning for the continuous assessment of BP. However, this method did not yield the required accuracy for clinical practice due to the respiratory variability of the ART signal.

Pulse transit time (PTT) has been known to be related to BP [2]. PTT is considered as the time delay for a pressure pulse to travel through a segment of an artery. Figure 1 shows the PTT [7] between an arterial line inserted in the radial artery and the PPG signal taken from a finger.

The relation between PTT and BP has been studied for decades and many models have been proposed to study this relation. Mukkamala et al. [2] provide a very good overview of the models available. Some general conclusions can be drawn from the literature available for the assessment of BP from PTT:

1. All PTT models require calibration with a cuff.

2. Most PTT models assess BP with a regression model, taking as input one of the following:

- PTT

- $1 / \mathrm{PTT}$ 
- $\log (\mathrm{PTT})$

- $1 /(\mathrm{PTT}-\mathrm{K})^{2}$

3. The parameters in the regression model depend on the assumptions made about the underlying model (i.e., geometry, flow, viscosity, elasticity, resistance, and so on).

In this short paper, we propose to study the feasibility of implementing a data-driven model from a calibration and PTT. The proposed data-driven model builds upon the method proposed in Ruiz-Rodríguez et al. [6], which takes advantage of an important property of artificial neural networks (ANN). This property states that ANN can be considered from a mathematical point of view as universal approximators. The universal approximation theorem states that a feed-forward network with a single layer containing a finite number of neurons can approximate continuous functions on compact subsets, under mild assumptions on the activation function. In plain language, this theorem implies that simple neural networks can represent a wide variety of functions when given the appropriate parameters. This property is particularly relevant for the problem at hand due to the fact that BP inference is very closely related to the Navier-Stokes equation, which lacks a general solution. Indeed, the solution of this equation remains an open problem in mathematical research. Therefore, the use of neural networks is better suited for the problem at hand due to the fact that the linear regression models normally used fail to deal with the nonlinear nature of BP inference.

In this paper, we shall also use the restricted Boltzmann machine (RBM) as the main building block for our regression model. The rationale behind using the RBM is that they are the simplest units to develop deep learning algorithms and there are also very efficient algorithms available for training deep architectures with these RBMs.

This study is meant to provide a first proof of concept for the validity and viability of a method for BP prediction based on RBM ANN models. The remaining of the paper is structured as follows: first, the dataset under analysis is summarily described. This is followed by a presentation of the validation and analysis methodologies. Then, results of the preliminary experiments are presented, some conclusions are drawn, and potential future lines of research are briefly outlined.

\section{Methods}

\section{Dataset Description}

The MIMIC II [8] database has been collected over a 17-year period beginning in 2001 from Boston's Beth Israel Deaconess
Medical Center (BIDMC), as part of a Bioengineering Research Partnership (BRP) grant. The project was formally established in 2003 including an interdisciplinary team from MIT, industry (Philips Medical Systems) and clinical medicine (BIDMC) with the objective of developing and evaluating advanced intensive care unit patient monitoring systems that would substantially improve the efficiency, accuracy, and timeliness of clinical decision making in intensive care. The requirement for individual patient consent was waived as the study did not impact clinical care and all data were de-identified.

For this proof of concept, we accessed the MIMIC II database in December 2015 and extracted the ART, ECG, and PPG records for 250 different patients. All biomedical signals were sampled at 125 samples/s. Biomedical signals were separated into 5 -s frames and a calibration from the ART signal was taken only for the first frame. After removing movement artifacts and noisy segments, we obtained a dataset with a total of 35,188 frames.

\section{Validation Methodology}

For the validation of our proposed approach, we have used the IEEE 1708-2014 standard for wearable, cuffless BP measuring devices. This standard divides the validation into two separate phases. In the first phase, at least 20 patients are recruited, whilst the second phase requires the recruitment of at least 25 extra patients. The main validation procedure is divided into three levels: static test, test with BP change from calibration point, and a test after a certain period of time from calibration.

The standard requires that $20 \%$ of the recordings/patients for phase I validation should fall in the normal range ( $\mathrm{SBP}<120 \mathrm{~mm}$ $\mathrm{Hg}$ and $\mathrm{DBP}<80 \mathrm{~mm} \mathrm{Hg}$ ), prehypertension (SBP between 120 and $139 \mathrm{~mm} \mathrm{Hg}$ and DBP between 80 and $89 \mathrm{~mm} \mathrm{Hg}$ ), stage 1 hypertension (SBP between 140 and $160 \mathrm{~mm} \mathrm{Hg}$ and DBP between 90 and $100 \mathrm{~mm} \mathrm{Hg}$ ), and stage 2 hypertension (SBP > $160 \mathrm{~mm} \mathrm{Hg}$ and DBP $>100 \mathrm{~mm} \mathrm{Hg}$ ). In order to comply with this requirement, our dataset has been scaled accordingly. At this point, it is also important to note that, in our case, we do not have a cuff-based calibration. For this reason, we have taken as both reference and calibration the BP values obtained from the ART signal.

Attending to the nature of our dataset, we shall report the results for the static test and a validation 6 min after calibration.

\section{Analysis Methodology and Data-Driven Model}

In our proposed model, we have taken as inputs the PTT calculated from the QRS complex of the first lead of the ECG recording and the PPG signal using the foot-to-foot algorithm presented in Gaddum et al. [7], which calculates the transit time as the delay between the valleys of waveforms at early systole. After this preprocessing, an RBM ANN with an architecture consisting of 3 layers (one input layer and two hidden layers) and 10 units (RBMs) for each layer has been trained with 25,150 frames from the dataset to obtain the BP values from our PTT inputs. The rest of frames have been reserved for testing. The learning rate (i.e., the hyperparameter that controls how much we are adjusting the weights of our network) was set to 0.001 whilst the learning rate for the hidden units was set to 0.002 . At this stage, it is important to note that learning rates must be kept small to guarantee the convergence of the learning algorithm and also improve the generalization capability of the resulting model. All networks have used pretraining with 10 epochs, and our RBMs also used weight decay and momentum term. 


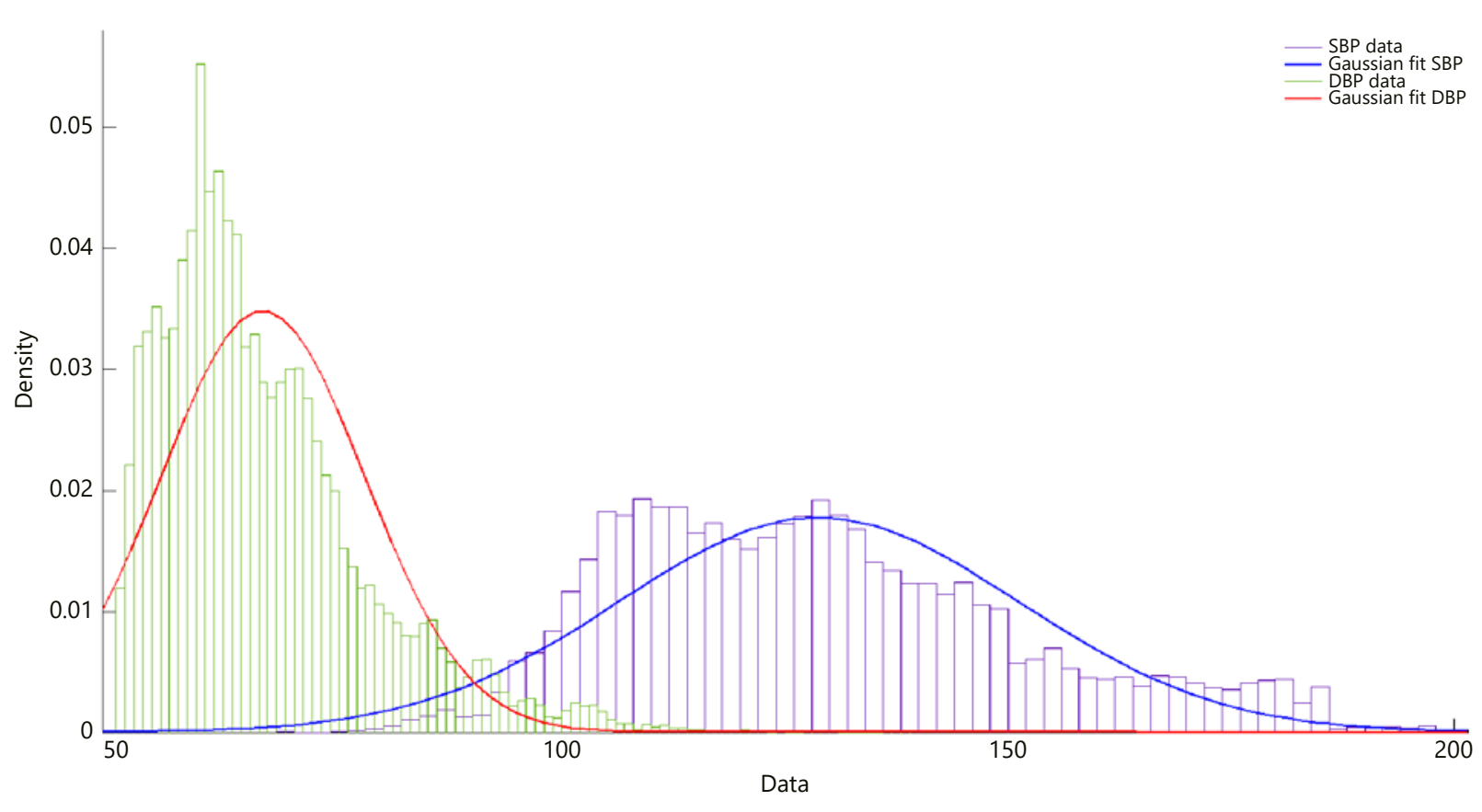

Fig. 2. Reference distributions and gaussian fit.

Table 1. Static test results

\begin{tabular}{llll}
\hline Model & BP & MAD & $\begin{array}{l}\text { Grading } \\
\text { (IEEE) }\end{array}$ \\
\hline RBM & SBP & $3.70 \mathrm{~mm} \mathrm{Hg}$ & $\mathrm{A}$ \\
Regression with PTT & SBP & $4.04 \mathrm{~mm} \mathrm{Hg}$ & B \\
Regression with 1/PTT & SBP & $3.47 \mathrm{~mm} \mathrm{Hg}$ & B \\
Regression with log(PTT) & SBP & $3.92 \mathrm{~mm} \mathrm{Hg}$ & B \\
RBM & DBP & $1.01 \mathrm{~mm} \mathrm{Hg}$ & A \\
Regression with PTT & DBP & $2.87 \mathrm{~mm} \mathrm{Hg}$ & A \\
Regression with 1/PTT & DBP & $2.87 \mathrm{~mm} \mathrm{Hg}$ & A \\
Regression with log(PTT) & DBP & $2.86 \mathrm{~mm} \mathrm{Hg}$ & A \\
\hline
\end{tabular}

\section{Results}

In compliance with the IEEE 1708-2014 standard, accuracy has been evaluated through the mean absolute difference (MAD). In particular,

$$
M A D=\sum_{i=1}^{N} \frac{\left|p_{i}-y_{i}\right|}{n},
$$

where $p_{i}$ corresponds to the test device measurement of $\mathrm{BP}, y_{i}$ is the average of the adjacent two reference $\mathrm{BP}$ mea-
Table 2. Results 6 min after calibration

\begin{tabular}{llll}
\hline Model & BP & MAD & $\begin{array}{l}\text { Grading } \\
\text { (IEEE) }\end{array}$ \\
\hline RBM & SBP & $4.76 \mathrm{~mm} \mathrm{Hg}$ & B \\
Regression with PTT & SBP & $4.83 \mathrm{~mm} \mathrm{Hg}$ & B \\
Regression with 1/PTT & SBP & $4.91 \mathrm{~mm} \mathrm{Hg}$ & B \\
Regression with log(PTT) & SBP & $4.83 \mathrm{~mm} \mathrm{Hg}$ & B \\
RBM & DBP & $3.41 \mathrm{~mm} \mathrm{Hg}$ & $\mathrm{A}$ \\
Regression with PTT & DBP & $3.52 \mathrm{~mm} \mathrm{Hg}$ & $\mathrm{A}$ \\
Regression with 1/PTT & DBP & $3.54 \mathrm{~mm} \mathrm{Hg}$ & $\mathrm{A}$ \\
Regression with $\log (\mathrm{PTT})$ & DBP & $3.52 \mathrm{~mm} \mathrm{Hg}$ & $\mathrm{A}$ \\
\hline
\end{tabular}

surements taken before and after the device measurement [8], and $n$ is the data size. Figure 2 presents the data distributions used in validation to calculate the MAD for DBP and SBP.

Tables 1 and 2 summarize the results obtained for our model, along with the grading given by the IEEE standard for our proposed model and three regression models used in the state of the art taking as inputs the PTT, 1/PTT and $\log (\mathrm{PTT})$. 


\section{Discussion and Conclusions}

In this short paper, we have studied the viability of implementing a noninvasive algorithm for the assessment of $\mathrm{BP}$ with a data-driven model obtained with RBMs. A first phase validation of our method shows a slight improvement over the state-of-the-art methods based on regression models over PTT. As it was expected, the performance of our regression model decreases as the measurement window separates from the calibration point. In our case, we had to evaluate 6 min after calibration. We consider that this decrease in performance is definitely a limitation for the use of these systems in a real clinical setting.

Another limitation of our study is that we had to take as a reference the $\mathrm{BP}$ values recorded from an arterial line instead of the noninvasive BP recording taken from a cuff, as required by the IEEE standard. However, we also believe that the results presented here are more pessimistic than those that would have been obtained with a cuff. As we have noted above, a limitation of cuff-based BP readings is that they tend to overestimate DBP and underestimate SBP.

Finally, a word of caution must be given. In our implementation, we have used data from 250 patients and, for testing purposes, we have scaled the test frames to fulfil the IEEE standard. We believe that this may limit the generalization capabilities of the proposed approach.
As future work, and in the light of the promising results obtained for this first evaluation phase, we propose to evaluate the capability of our system to track sudden changes in BP as required by the IEEE standard over a larger population. In this evaluation, we could also try to implement a regression model over a recurrent neural network to further improve the tracking capabilities of the end system.

\section{Statement of Ethics}

Ethics committee approval was not required and the requirement for individual patient consent was waived as the MIMIC II study did not impact clinical care and all data were de-identified.

\section{Disclosure Statement}

The authors declare that they have no conflicting interests for the publication of this article.

\section{Funding Sources}

This research was funded by the Catalan Agency ACCIÓ through the Health Forecast Initiative and by Spanish MINECO TIN2016-79576-R research project.

\section{References}

1 Warren DK, Quadir WW, Hollenbeak CS, Elward AM, Cox MJ, Fraser VJ: Attributable cost of catheter-associated bloodstream infections among intensive care patients in a nonteaching hospital. Crit Care Med 2006;34: 2084-2089.

2 Mukkamala R, Hahn JO, Inan OT, Mestha LK, Kim CS, Töreyin H, Kyal S: Toward ubiquitous blood pressure monitoring via pulse transit time: theory and practice. IEEE Trans Biomed Eng 2015;62:1879-1901.

3 Chemla D, Teboul JL, Richard C: Noninvasive assessment of arterial pressure. Curr Opin Crit Care 2008;14:317-321.
4 Bur A, Hirschl MM, Herkner H, Oschatz E, Kofler J, Woisetschläger C, Laggner AN: Accuracy of oscillometric blood pressure measurement according to the relation between cuff size and upper-arm circumference in critically ill patients. Crit Care Med 2000;28: 371-376.

5 Jones DW, Apple LJ, Sheps SG, Roccella EJ, Lenfant C: Measuring blood pressure accurately: new and persistent changes. JAMA 2003;289:1027-1030.
6 Ruiz-Rodríguez JC, Ruiz-Sanmartín A, Ribas V, Caballero J, García-Roche A, Riera J, et al: Innovative continuous non-invasive cuffless blood pressure monitoring based on photoplethysmography technology. Intensive Care Med 2013;39:1618-1625.

7 Gaddum NR, Alastruey J, Beerbaum P, Chowienczyk P, Schaeffter T: A technical assessment of pulse wave velocity algorithms applied to non-invasive arterial waveforms. Ann Biomed Eng 2013;41:2617-2629.

8 Saeed M, Lieu C, Raber G, Mark RG: MIMIC II: a massive temporal ICU patient database to support research in intelligent patient monitoring, Comput Cardiol 2002;29:641-644. 